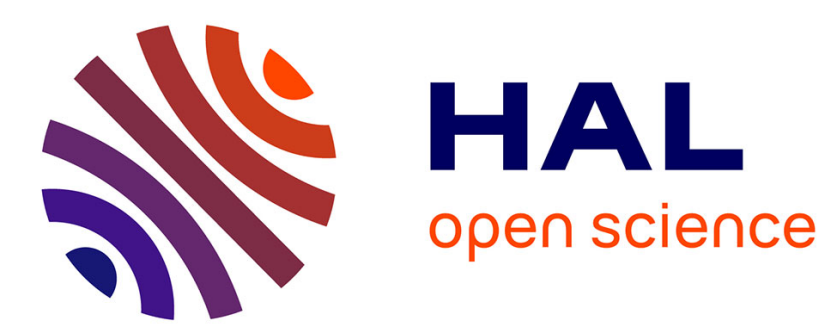

\title{
Elliptic Cliffordian Functions
}

Guy Laville, Ivan Ramadanoff

\section{- To cite this version:}

Guy Laville, Ivan Ramadanoff. Elliptic Cliffordian Functions. Complex Variables, 2001, 45, pp.4,297-

318. hal-00003214

\section{HAL Id: hal-00003214 \\ https://hal.science/hal-00003214}

Submitted on 3 Feb 2005

HAL is a multi-disciplinary open access archive for the deposit and dissemination of scientific research documents, whether they are published or not. The documents may come from teaching and research institutions in France or abroad, or from public or private research centers.
L'archive ouverte pluridisciplinaire HAL, est destinée au dépôt et à la diffusion de documents scientifiques de niveau recherche, publiés ou non, émanant des établissements d'enseignement et de recherche français ou étrangers, des laboratoires publics ou privés. 


\title{
ELLIPTIC CLIFFORDIAN FUNCTIONS
}

by

Guy Laville and Ivan Ramadanoff

\begin{abstract}
In the study of holomorphic functions of one complex variable, one well-known theory is that of elliptic functions and it is possible to take the $\zeta$-function of Weierstrass as a building stone of this vast theory. We are working the analogue theory in the natural context of higher dimensional spaces : holomorphic and elliptic Cliffordian functions.
\end{abstract}

\section{$\oint 1$. Elliptic Cliffordian functions}

Everywhere in this paper we will be interested in functions $f: \Omega \rightarrow \mathbb{R}_{0,3}$, where $\Omega$ is an open subset of $\mathbb{R}^{4}$. In previous papers $[8,9]$ we introduced the notion of (left) holomorphic Cliffordian function acting from an open subset of $\mathbb{R}^{2 m+2}$ in the Clifford algebra $\mathbb{R}_{0,2 m+1}, m \in \mathbb{N}$.

Let us recall the basic definition and some general properties of holomorphic Cliffordian functions. Consider the Clifford algebra $\mathbb{R}_{0,2 m+1}$ of the real vector space $V$ of dimension $2 m+1$, provided with a quadratic form of negative signature. Denote by $S$ the set of the scalars in $\mathbb{R}_{0,2 m+1}$ which can be identified to $\mathbb{R}$. Let $\left\{e_{i}\right\}, i=1,2, \ldots, 2 m+1$ be an orthonormal basis of $V$ and let $e_{0}=1$. Thus, $\left\{e_{0}, e_{1}, \ldots, e_{2 m+1}\right\}$ would be a basis for the algebra $\mathbb{R}_{0,2 m+1}$ such that $e_{i} e_{j}+e_{j} e_{i}=-2 \delta_{i j}$ for $0 \leq i, j \leq 2 m+1$ and $\delta_{i j}$ is the Kronecker symbol.

Definition.- Given an open subset $\Omega$ of $S \oplus V$, a function $f: \Omega \rightarrow \mathbb{R}_{0,2 m+1}$ is said to be (left) holomorphic Cliffordian on $\Omega$ if and only if :

$$
D \Delta^{m} f(x)=0
$$

for each $x$ of $\Omega$. Here $\Delta^{m}$ means the iterated $m$ times Laplacian $\Delta$ and $D$ is the usual operator for monogenic functions [1], [3], [4] :

$$
D=\sum_{i=0}^{2 m+1} e_{i} \frac{\partial}{\partial x_{i}} .
$$


It is clear that, in the case when $m=0$, such a function would be an usual holomorphic function of one complex variable.

Looking at the previous definition, one could remark that, because of the appearance of the Laplacian, a kind of qualitative jump takes place when the integer $m$ passes from 0 to 1 . In fact, there would be no major differences in the studies of holomorphic Cliffordian functions when $m \geq 1$. That is the reason, and also in order to have lighter written expressions of our formulas, we will restrict us to the case $m=1$.

Referring again to $[8,9]$, let us recall that holomorphic Cliffordian functions verify an integral representation formula which can be looked as an analogous of the Cauchy integral formula for holomorphic functions. Thus, we get :

Theorem. (Taylor expansion).- Let $f$ be a holomorphic Cliffordian function in an open neighborhood $W$ of $a \in S \oplus V$. Then, for each $x \in W$ :

$$
f(x)=\sum_{k=1}^{\infty} \sum_{|\alpha|=k} P_{\alpha}(x-a) c_{\alpha}
$$

were $\alpha=\left(\alpha_{0}, \alpha_{1}, \alpha_{2}, \alpha_{3}\right)$ is a multiindex of $\mathbb{N}^{4},|\alpha|$ denotes its length $|\alpha|=$ $\alpha_{0}+\alpha_{1}+\alpha_{2}+\alpha_{3}, \quad c_{\alpha} \in \mathbb{R}_{0,3}$ and the $P_{\alpha}$ are left (and right) holomorphic Cliffordian polynomials of degree $|\alpha|-1$ on $x$ defined as follows : consider the set $\left\{e_{\nu}\right\}=\left\{e_{0}, \ldots, e_{0}, e_{1}, \ldots, e_{1}, e_{2}, \ldots, e_{2}, e_{3}, \ldots, e_{3}\right\}$ where $e_{0}$ is written $\alpha_{0}$ times, $e_{1}: \alpha_{1}$ times, etc $\ldots$

Set :

$$
P_{\alpha}(x)=\sum_{\mathfrak{S}} \prod_{\nu=1}^{|\alpha|-1}\left(e_{\sigma(\nu)} x\right) e_{\sigma(|\alpha|)}
$$

the sum being expanded over all distinguishable elements $\sigma$ of the permutation group $\mathfrak{S}$ of the set $\left\{e_{\nu}\right\}$.

It should be noted that, for a fixed $k \in \mathbb{N}^{*}$, the set $\left\{P_{\alpha}:|\alpha|=k\right\}$ contains $C_{k+3}^{3}$ polynomials. One could also remark that there is a generating function for the polynomials $P_{\alpha}$. Indeed, if we set :

$$
\lambda=\sum_{i=0}^{4} \lambda_{i} e_{i}, \quad \lambda_{i} \in \mathbb{R} \quad \text { and } \quad \lambda_{\alpha}=\prod_{i=0}^{4} \lambda_{i}^{\alpha_{i}},
$$

then the following formal series gives a generating function :

$$
(1-\lambda x)^{-1} \lambda=\sum_{\alpha} P_{\alpha}(x) \lambda_{\alpha}
$$

Using similar notations, set $\beta \in \mathbb{N}^{4}$ a multiindex of length $|\beta|$. Consider the same set $\left\{e_{\nu}\right\}$ as before and put :

$$
S_{\beta}(x)=\sum_{\mathfrak{S}} \prod_{\nu=1}^{|\beta|}\left(x^{-1} e_{\sigma(\nu)}\right) x^{-1} .
$$


Thus we will have for example $S_{(1,0,0,0)}(x)=x^{-1} e_{0} x^{-1}, \quad S_{(0,1,1,0)}(x)=$ $x^{-1} e_{1} x^{-1} e_{2} x^{-1}+x^{-1} e_{2} x^{-1} e_{1} x^{-1}$. In the rational function $S_{\beta}$ the power of $x^{-1}$ is $|\beta|+1$. It is not difficult to observe that the $S_{\beta}$ are generated by $x^{-1}$ through derivation and thus the $S_{\beta}$ are left (and right) holomorphic Cliffordian function excepting at 0 . This allows us to deduce :

Theorem. (Laurent expansion).- If $B$ is a ball in $S \oplus V$ centered at the origin and if $f: B \backslash\{0\} \rightarrow \mathbb{R}_{0,3}$ is a holomorphic Cliffordian function, then for each $x \in B \backslash\{0\}$ :

$$
f(x)=\sum_{|\beta|=0}^{\infty} S_{\beta}(x) d_{\beta}+\sum_{|\alpha|=1}^{\infty} P_{\alpha}(x) c_{\alpha},
$$

where $c_{\alpha}$ and $d_{\beta}$ belong to $\mathbb{R}_{0,3}$.

In such a way, the first sum should be considered as the analogous of the singular part of a Laurent expansion for a holomorphic function, while the second sum represents the analogous of its regular part.

Let us make some additional remarks. If $f$ is a real analytic function on a neighborhood $W$ of $a \in S \oplus W$ and taking its values in $S \oplus V$, we can look at $f$ as a $C^{\infty}$ application of $W \subset \mathbb{R}^{4}$ into $\mathbb{R}^{4}$ so that $f$ possesses an usual Taylor expansion which can be written as :

$$
f(a+h)=\left.\sum_{n=0}^{\infty} \frac{1}{n !}\left(h \mid \nabla_{x}\right)^{n} f(x)\right|_{x=a}
$$

where $\left(h \mid \nabla_{x}\right)$ is the usual scalar product in $\mathbb{R}^{4}$ of the two vectors $h=\left(h_{0}, h_{1}, h_{2}, h_{3}\right)$ and the gradient $\nabla_{x}=\left(\frac{\partial}{\partial x_{0}}, \frac{\partial}{\partial x_{1}}, \frac{\partial}{\partial x_{2}}, \frac{\partial}{\partial x_{3}}\right)$ applied on $f$ as a function of $x=\left(x_{0}, x_{1}, x_{2}, x_{3}\right) ;(h \mid \nabla)^{n}$ means $(h \mid \nabla) \cdots(h \mid \nabla)$ repeated $n$ times and, of course $a+h \in S \oplus V$.

They are some formal calculations related to the operator $\left(h \mid \nabla_{x}\right)$ which should be noted. For instance : $\left(h \mid \nabla_{x}\right)(x)=h$ and since $x x^{-1}=1$, then $\left(h \mid \nabla_{x}\right)\left(x x^{-1}\right)=0$, but also

$$
h x^{-1}+x\left(h \mid \nabla_{x}\right)\left(x^{-1}\right)=0
$$

so that :

$$
\left(h \mid \nabla_{x}\right)\left(x^{-1}\right)=-x^{-1} h x^{-1} .
$$

By iteration on $q \in \mathbb{N}$, one get :

$$
\left(h \mid \nabla_{x}\right)^{q}\left(x^{-1}\right)=(-1)^{q} q !\left(x^{-1} h\right)^{q} x^{-1} .
$$


Remember that the statement of the classical theorem of Liouville for holomorphic functions remains valid for harmonic functions and also for monogenic functions $([1]$, p.99). This allows us to state the following :

Theorem of Liouville.- If $f$ is a holomorphic Cliffordian function in the whole open set $S \oplus V$, identified to $\mathbb{R}^{4}$, (such a function could be called entire Cliffordian) and if $f$ and its derivatives up to the second order are bounded, then $f$ reduces to a constant.

Whitout loss of generality we may achieve the proof for all the components of $f$ taking its values in $\mathbb{R}_{0,3}$, so in what follows we may assume that $f: \mathbb{R}^{4} \rightarrow \mathbb{R}$. Set $g=\Delta f$. Thus $g$ is monogenic and the Liouville theorem for monogenic functions says that $g$ reduces to a constant, i.e. $\Delta f=K, K \in \mathbb{R}$. Remark the condition $g$ bounded is fulffiled because of the boundedness of the derivatives of $f$. But $\Delta f=K$ implies $\Delta\left(\frac{\partial f}{\partial x_{i}}\right)=0$, $i=0,1,2,3$. Apply now the Liouville theorem for harmonic functions, one deduces $\frac{\partial f}{\partial x_{i}}=c_{i}, \quad i=0,1,2,3, \quad c_{i} \in \mathbb{R}$. This means that

$$
f\left(x_{0}, x_{1}, x_{2}, x_{3}\right)-\sum_{i=0}^{3} c_{i} x_{i}
$$

satisfies $d f=0$, from which we get that $f$ reduces to a polynomial on $x_{0}, x_{1}, x_{2}, x_{3}$ of first degree. Remember again $f$ must be bounded, so that $f$ reduces to a constant.

It should be noted that the same argument allows to get a Liouville theorem for biharmonic functions under the assumption such a biharmonic function should be bounded with all its derivatives up to the second order.

Remark that all we said on the holomorphic Cliffordian functions, including their Laurent expansions, allows us to consider meromorphic Cliffordian functions in the sense that such a function would be holomorphic Cliffordian in an open subset $\Omega$ of $S \oplus V$, excepting at isolated pointwise singularities, namely pointwise poles, i.e.such that the principal part of their Laurent expansion contains only a finite number of terms. So, put :

Definition.- A function $f: \Omega \rightarrow \mathbb{R}_{0,3}$, where $\Omega$ is an open subset of $S \oplus V$ is said to be meromorphic Cliffordian if $f$ is holomorphic Cliffordian on $\Omega$, excepting on an isolated set of pointwise poles.

In this sense $x^{-1}$ is a meromorphic function in $S \oplus V$ with a simple pole at the origin. 
The variety of singularities of general meromorphic functions is larger. As the following example shows, the set of singularities of a Cliffordian function might be quite complicated :

Look at $g: x \longmapsto\left(x_{1}-e_{1} x_{0}\right)^{-1}$, which is holomorphic Cliffordian everywhere except at the subset $\left\{x_{1}=0, x_{0}=0\right\}$ of $\mathbb{R}^{4}$.

The study of the singularities sets which are not pointwise deserves to be accomplished but will not be the subject of this paper.

We will end this paragraph putting the basis of elliptic Cliffordian function theory. As in the case of functions of a complex variable, where they are two kinds of periodic functions - the 1-periodic and the doubly periodic functions, here we could expect much more subclasses of periodic functions, namely the $N$-periodic functions, where $N=1,2,3,4$.

So take $N$ in $\{1,2,3,4\}$. Let $\omega_{\alpha} \in S \oplus V$ for $\alpha=1,2, \ldots, N$. The $\omega_{\alpha}$ will play the role of half periods. We will always suppose the paravectors $\omega_{1}, \ldots, \omega_{N}$ linearly independant in $S \oplus V$.

Definition.- A function $f: S \oplus V \rightarrow \mathbb{R}_{0,3}$ is said to be $N$-periodic if

for every $x \in S \oplus V$.

$$
f\left(x+2 \omega_{\alpha}\right)=f(x)
$$

Introduce $\omega=\left(\omega_{1}, \ldots, \omega_{N}\right)$ and, for a multiindex $k=\left(k_{1}, \ldots, k_{N}\right)$ belonging to $\mathbb{Z}^{N}$, define $k \omega$ by $k \omega=\sum_{\alpha=1}^{N} k_{\alpha} \omega_{\alpha}$. Let us call the set $2 \mathbb{Z}^{N} \omega=\left\{2 k \omega, k \in \mathbb{Z}^{N}\right\}$ a lattice. A fundamental cell of this lattice will be the hypercube spanned over $2 \omega_{1}, 2 \omega_{2}, 2 \omega_{3}, 2 \omega_{4}$.

Obviously, for a $N$-periodic function $f$, we have :

$$
f(x+2 k \omega)=f(x) .
$$

Now, define :

Definition.- A function $f: S \oplus V \rightarrow \mathbb{R}_{0,3}$ is said elliptic Cliffordian if $f$ is $N$-periodic and meromorphic.

The first fundamental result of the classical theory of elliptic functions says that an elliptic function which is holomorphic reduces to a constant. Here we have the same : 
Theorem 1.- An elliptic Cliffordian function which is holomorphic Cliffordian reduces to a constant.

Indeed, a holomorphic Cliffordian function is obviously bounded on a fundamental cell and by periodicity would be bounded on the whole espace $S \oplus V$. The same is true for all its derivatives because if $f$ is $N$-periodic, then all the $\left(e_{i} \mid \nabla_{x}\right) f(x)$ are also $N$-periodic. All of them would be bounded on $S \oplus V$ via the fundamental cell. It remains just to apply the Liouville's theorem and to conclude that $f$ reduces to a constant.

It should be remarked that the present theory of elliptic Cliffordian function is only an additive theory in the sense that elliptic Cliffordian functions could be added and multiplied (in $\mathbb{R}_{0,3}$ ) by Clifford constants.

At this time we do not dispose with a satisfactory definition of a product of two elliptic Cliffordian functions.

The next theorem is an example of what we can do only with additive arguments :

Theorem 2.- If $f_{1}$ and $f_{2}$ are two elliptic Cliffordian functions with the same pointwise poles and the same principal part of their Laurent expansions on the neighborhoods of their poles, then they differ just up to an additive constant.

The proof is easy : consider $f_{1}-f_{2}$. This is a holomorphic Cliffordian function which is periodic and by theorem 1 must be a constant.

\section{$\S 2$. On the trigonometric Cliffordian functions}

Let us start with a slight modification of the lemma of $\S 4$ in [9] which says :

LEMMA 1.- If $u: \mathbb{R}^{2} \rightarrow \mathbb{R},(\xi, \eta) \longmapsto u(\xi, \eta)$ is harmonic, then $\mathcal{U}(x)=$ $u\left(x_{0}, \vec{x}\right)$, where $x=x_{0}+\vec{x}$, is biharmonic, i.e. $\Delta^{2} \mathcal{U}(x)=0$.

With the help of this lemma, we dispose a way to generate holomorphic Cliffordian functions, taking $D^{*} \mathcal{U}$. Recall that :

$$
D=\frac{\partial}{\partial x_{0}}+\sum_{i=1}^{3} e_{i} \frac{\partial}{\partial x_{i}}
$$


and $D^{*}=\frac{\partial}{\partial x_{0}}-\sum_{i=1}^{3} e_{i} \frac{\partial}{\partial x_{i}}$, so that if we introduce the notations $D_{0}=\frac{\partial}{\partial x_{0}}$ and $\vec{D}=\sum_{i=1}^{3} e_{i} \frac{\partial}{\partial x_{i}}$, we have $D=D_{0}+\vec{D}$ and $D^{*}=D_{0}-\vec{D}$.

Now, let us modify the above lemma in order to have a way for generating holomorphic Cliffordian functions directly from the holomorphic ones :

LEMMA 2.- If $f:(\xi, \eta) \longmapsto f=u+i v$ is a holomorphic function, then $F(x)=u\left(x_{0},|\vec{x}|\right)+\frac{\vec{x}}{|\vec{x}|} v\left(x_{0},|\vec{x}|\right) \quad$ is a holomorphic Cliffordian function.

Proof.- Given $f$ holomorphic, there exists $h$, which is harmonic and $2 \frac{\partial}{\partial z} h=f$, so that $\frac{\partial h}{\partial \xi}=u, \frac{\partial h}{\partial \eta}=-v$. Apply lemma 1 to $h$, we construct $H(x)=h\left(x_{0},|\vec{x}|\right)$ which will be biharmonic and thus $F(x)=D^{*} H(x)$ should be holomorphic Cliffordian. But :

$$
D^{*} H(x)=D_{0} H(x)-\vec{D} H(x) .
$$

However,

$$
\begin{aligned}
& D_{0} H(x)=\frac{\partial h}{\partial x_{0}}\left(x_{0},|\vec{x}|\right)=\left.\frac{\partial h}{\partial \xi}(\xi, \eta)\right|_{\xi=x_{0}, \eta=\mid \vec{x}}=u\left(x_{0},|\vec{x}|\right), \text { and } \\
& \vec{D} H(x)=\left.\frac{\partial h}{\partial \eta}(\xi, \eta)\right|_{\xi=x_{0}, \eta=|\vec{x}|} \cdot \vec{D}(|\vec{x}|), \text { and } \\
& \vec{D}(|\vec{x}|)=\vec{D}\left(\left(|\vec{x}|^{2}\right)^{\frac{1}{2}}=\frac{1}{2} \frac{1}{|\vec{x}|} \vec{D}\left(|\vec{x}|^{2}\right)=\frac{\vec{x}}{|\vec{x}|},\right.
\end{aligned}
$$

so that $\vec{D} H(x)=-\frac{\vec{x}}{|\vec{x}|} v\left(x_{0},|\vec{x}|\right)$ and finally :

$$
D^{*} H(x)=F(x)=u\left(x_{0},|\vec{x}|\right)+\frac{\vec{x}}{|\vec{x}|} v\left(x_{0},|\vec{x}|\right) .
$$

Lemma 2 is a fruitful tool for generating the analogous of the trigonometric functions $\sin z, \cos z$ etc ... in the frame of Cliffordian analysis.

Thus, for example, the function

$$
e^{x}=e^{x_{0}}\left(\cos \sqrt{-\vec{x}^{2}}+\frac{\vec{x}}{|\vec{x}|} \sin \sqrt{-\vec{x}^{2}}\right)
$$


is a well-defined holomorphic Cliffordian function from $S \oplus V$ in $S \oplus V \subset$ $\mathbb{R}_{0,3}$.

Further,

$$
\begin{aligned}
& \sin x=-\frac{\vec{x}}{|\vec{x}|} \frac{e^{x}-e^{-x}}{2}, \\
& \cos x=\frac{e^{x}+e^{-x}}{2}, \\
& \operatorname{cotan} x=\frac{\vec{x}}{|\vec{x}|} \frac{e^{x}+e^{-x}}{e^{x}-e^{-x}} .
\end{aligned}
$$

Lemma 2 allows us to consider all of them as holomorphic Cliffordian functions. The final procedure for constructing such functions is to make a formal change of the letters $z$ in $x, \quad \xi=\mathcal{R} e\{z\}$ in $x_{0}, \quad i \eta=i \Im\{z\}$ in $\vec{x}$, or equivalently $i$ in $\frac{\vec{x}}{|\vec{x}|}$ and $\eta$ in $|\vec{x}|$.

\section{Weierstrass $\zeta$ holomorphic Cliffordian functions}

Remember that the point of view of Weierstrass theory of elliptic functions (doubly-periodic and meromorphic) is based on the $\mathcal{P}$-function which appears naturally as the derivative, up to the sign, of the $\zeta$ - function of Weierstrass.

In this paragraph we will construct four $\zeta_{N}$ Weierstrass functions in $\mathbb{R}_{0,3}$. Take again $N$ in $\{1,2,3,4\}$. Introduce $\omega=\left(\omega_{1}, \ldots, \omega_{N}\right)$ where $\omega_{\alpha} \in$ $S \oplus V$ for $\alpha=1,2, \ldots, N$, and suppose the paravectors $\omega_{1}, \ldots, \omega_{N}$ linearly independant in $S \oplus V$. For a multiindex $k=\left(k_{1}, \ldots, k_{N}\right)$ belonging to $\mathbb{Z}^{N}$, define $k \omega$ by $k \omega=\sum_{\alpha=1}^{N} k_{\alpha} \omega_{\alpha}$. Taking into account the countability of the respective sets, it is not difficult to rearrange the lattice $2 \mathbb{Z}^{N} \omega \backslash\{(0, \ldots, 0)\}$ as $\left\{w_{p}\right\}_{p=1}^{\infty}$.

Definition.- A Weierstrass $\zeta_{N}$-function with lattice $2 \mathbb{Z}^{N} \omega$ is the function $\zeta_{N}: S \oplus V \backslash 2 \mathbb{Z}^{N} \omega \rightarrow \mathbb{R}_{0,3}$ defined by :

$$
\zeta_{N}(x)=x^{-1}+\sum_{p=1}^{\infty}\left\{\left(x-w_{p}\right)^{-1}+\sum_{\mu=0}^{N-1}\left(w_{p}^{-1} x\right)^{\mu} w_{p}^{-1}\right\} .
$$

In such a way we get four functions $\zeta_{1}, \zeta_{2}, \zeta_{3}, \zeta_{4}$ which are related respectively with the 1 -periodical lattice $\left\{2 k_{1} \omega_{1}, k_{1} \in \mathbb{Z}, \omega_{1} \in S \oplus V\right\}$ for the first one and with a 4-periodical lattice for the last one. 
Consider a particular case concerning $\zeta_{1}$, setting $\omega_{1}=\frac{\pi}{2}$ :

$$
\zeta_{1}(x)=x^{-1}+\sum_{k_{1} \in \mathbb{Z}^{*}}\left\{\left(x-k_{1} \pi\right)^{-1}+\left(k_{1} \pi\right)^{-1}\right\} .
$$

Thus we recognize the analogous of the function $\operatorname{cotg} z$ in the function theory of one complex variable.

Remark also that $\zeta_{2}$ is analogous to the well-known Weierstrass $\zeta$ function of a complex variable :

$$
\zeta(z)=\frac{1}{z}+\sum_{p=1}^{\infty}\left\{\frac{1}{z-w_{p}}+\frac{1}{w_{p}}+\frac{z}{w_{p}^{2}}\right\} .
$$

Here obviously, the subset $\left(2 \mathbb{Z} \omega_{1}+2 \mathbb{Z} \omega_{2}\right) \backslash\{(0,0)\}$ of the corresponding lattice has been rearranged in a set noted $\left\{w_{p}\right\}, p \in \mathbb{N}^{*}$.

So we have obtained four $\zeta$-functions. If we pursue in the way of the similarity with holomorphic functions, we could expect that all of them be holomorphic Cliffordian excepted at the points of the lattice. They probably would not be themselves $N$-periodic, but by $N$ derivations they would generated the analogous $\mathcal{P}_{N}$ of the Weierstrass $\mathcal{P}$-function.

First of all, note that the series

$$
\sum_{w \in \Omega_{N}, w \neq 0}|w|^{-N-1}
$$

converges where $\Omega_{N}$ designes a $N$-periodic lattice in $\mathbb{R}^{4}$. This is a result following easily from similar arguments as in the classical case.

The existences of the $\zeta_{N}$ will be deduced from the proof of the convergences of the series defining $\zeta_{N}$. For this, a majoration of the general term of the series will be achieved. First, remark the general term of the series could be written as :

$$
\begin{gathered}
\left(x-w_{p}\right)^{-1}+\sum_{\mu=0}^{N-1}\left(w_{p}^{-1} x\right)^{\mu} w_{p}^{-1}=\left\{1+\sum_{\mu=0}^{N-1}\left(w_{p}^{-1} x\right)^{\mu} w_{p}^{-1}\left(x-w_{p}\right)\right\}\left(x-w_{p}\right)^{-1}= \\
=\left\{\sum_{\nu=0}^{N}\left(w_{p}^{-1} x\right)^{\nu}-\sum_{\mu=0}^{N-1}\left(w_{p}^{-1} x\right)^{\mu}\right\}\left(x-w_{p}\right)^{-1}=\left(w_{p}^{-1} x\right)^{N}\left(x-w_{p}\right)^{-1} .
\end{gathered}
$$

Now, let us consider the compact set $K_{r}=\{x \in S \oplus V:|x| \leq r\}$. Thus, except for a finite number of $w_{p}$, one has $\left|w_{p}\right| \geq 2 r$. 
For $|x| \leq r$, the next majoration follows :

$$
\begin{gathered}
\left|\left(w_{p}^{-1} x\right)^{N}\left(x-w_{p}\right)^{-1}\right|=\left|\left(w_{p}^{-1} x\right)^{N}\left(w_{p}^{-1} x-1\right)^{-1} w_{p}^{-1}\right| \leq \\
\leq \frac{r^{N}}{2}\left|w_{p}^{-1}\right|^{N+1}=C_{N, r}\left|w_{p}\right|^{-N-1} .
\end{gathered}
$$

In such a way we proved that the series defining $\zeta_{N}$ are uniformly convergent on compact subsets of $S \oplus V$ for $N=1,2,3,4$.

It should be noted that the limit (or the sum) of every sequence (or series) of holomorphic Cliffordian functions converging uniformly on compact sets is also a holomorphic Cliffordian function. The reason is that $f$ is holomorphic Cliffordian if and only if $D D^{*} D f=0$, so that applying three times the corresponding theorem of Weierstrass for monogenic functions [1], p.58, we get the result.

In our case, all the terms in the series defining $\zeta_{N}$ are holomorphic Cliffordian in $S \oplus V$ excepting at the points $w_{p}$ and the convergence being uniform on compact subsets, we can say that $\zeta_{N}$ is a holomorphic Cliffordian function on $S \oplus V \backslash\left\{w_{p}\right\}_{p=0}^{\infty}$, where $w_{0}=(0,0, \ldots, 0)$, or equivalently on $S \oplus V \backslash 2 \mathbb{Z}^{N} \omega$.

Generally, a holomorphic Cliffordian function acts from $S \oplus V$ in $\mathbb{R}_{0,3}$. As far as the $\zeta_{N}$ functions are concerned, the first observation we can do, is that $\zeta_{N}$ possesses simple poles at the points of the lattice (in fact, by construction). We could remark also that $\zeta_{N}$ takes its values not in the whole Clifford algebra $\mathbb{R}_{0,3}$, but in a subset, which is exactly $S \oplus V$.

Indeed, $x^{-1}=\frac{x^{*}}{|x|^{2}}$, where $x^{*}$ is the conjugate of $x, x^{*}=x_{0} e_{0}-\sum_{j=1}^{3} x_{j} e_{j}$, obviously belongs to $S \oplus V$. A straightforward computation carried on $h x h$, where $x, h \in S \oplus V$ shows that $h x h \in S \oplus V$ and then, using a reccurrence argument, one can show that $(h x)^{n} h \in S \oplus V$ for $n \in \mathbb{N}$.

At the end of this paragraph let us see that the $\zeta_{N}$ functions are odd. In fact, there is another way to write down the expressions of the $\zeta_{N}$ functions, namely :

$$
\zeta_{N}(x)=x^{-1}-\sum_{n=N}^{\infty} \sum_{p=1}^{\infty}\left(w_{p}^{-1} x\right)^{n} w_{p}^{-1}
$$

which is easily obtained developping $\left(x-w_{p}\right)^{-1}$ as $-w_{p}^{-1}-w_{p}^{-1} x w_{p}^{-1}-$ $\cdots-\left(w_{p}^{-1} x\right)^{n} w_{p}^{-1}-\cdots$ in (1). Here, we have to remember that the set $\left\{w_{p}\right\}_{p=1}^{\infty}$ is exactly $2 \mathbb{Z}^{N} \omega \backslash\{(0, \ldots, 0)\}$. Therefore, the sum (2) contains terms of the type

$$
\left\{(2 k \omega)^{-1} x(2 k \omega)^{-1}\right\}^{n}(2 k \omega)^{-1}
$$


for $k \in \mathbb{Z}^{N} \backslash\{(0, \ldots, 0)\}$ and $n \geq N$. But when $n$ is even, we meet "opposite" vertices of the lattice, i.e. such that the lengths of the corresponding multiindices are $+|k|$ and $-|k|$ and thus the corresponding terms in the sum will cancelled. So the sum is expanded over those $n$ which are odd integers and we get

$$
\zeta_{N}(-x)=-\zeta_{N}(x)
$$

for $x \in S \oplus V \backslash\left\{w_{p}\right\}_{p=0}^{\infty}$ and $N=1,2,3,4$. The formula (2) is reduced to :

$$
\zeta_{N}(x)=x^{-1}-\sum_{k \geq\left[\frac{N}{2}\right]} \sum_{p=1}^{\infty}\left(w_{p}^{-1} x\right)^{2 k+1} w_{p}^{-1} .
$$

Remark this formula coïncides with the well know formula for the classical Weierstrass $\zeta$ function in the case $N=2$. That we obtained is :

$$
\begin{gathered}
\zeta_{2}(x)=x^{-1}-\sum_{k \geq 1} \sum_{p=1}^{\infty}\left(w_{p}^{-1} x\right)^{2 k+1} w_{p}^{-1}= \\
=x^{-1}-\sum_{p=1}^{\infty}\left(w_{p}^{-1} x\right)^{3} w_{p}^{-1}-\sum_{p=1}^{\infty}\left(w_{p}^{-1} x\right)^{5} w_{p}^{-1}-\cdots
\end{gathered}
$$

which is the analogous of :

$$
\zeta(z)=\frac{1}{z}-z^{3} \sum_{p=1}^{\infty} \frac{1}{w_{p}^{4}}-z^{5} \sum_{p=1}^{\infty} \frac{1}{w_{p}^{6}}-\cdots
$$

We will end this paragraph by the Laurent expansion of $\zeta_{N}$ in a neighborhood of the arign. This can be achieved shortly combining (2) with the last formula of $\S 1$ we could write as :

$$
(x \mid \nabla)^{n}\left(w_{p}^{-1}\right)=(-1)^{n} n !\left(w_{p}^{-1} x\right)^{n} w_{p}^{-1},
$$

where $x, w_{p} \in S \oplus V ; p, n \in \mathbb{N}$. So, we get :

$$
\begin{aligned}
\zeta_{N}(x) & =x^{-1}-\sum_{n=N}^{\infty} \sum_{p=1}^{\infty}(-1)^{n} \frac{(x \mid \nabla)^{n}}{n !}\left(w_{p}^{-1}\right) \\
& =x^{-1}-\sum_{n=N}^{\infty}(-1)^{n} \frac{(x \mid \nabla)^{n}}{n !}\left(\sum_{p=1}^{\infty} w_{p}^{-1}\right) .
\end{aligned}
$$

Taking into account (3), we have :

$$
\zeta_{N}(x)=x^{-1}+\sum_{k \geq\left[\frac{N}{2}\right]} \frac{(x \mid \nabla)^{2 k+1}}{(2 k+1) !}\left(\sum_{p=1}^{\infty} w_{p}^{-1}\right) .
$$


In this way we are able to give another form to the classical formula (3'), namely :

$$
\zeta(z)=\frac{1}{z}+\frac{(z \mid \nabla)^{3}}{3 !}\left(\sum_{p=1}^{\infty} \frac{1}{w_{p}}\right)+\frac{(z \mid \nabla)^{5}}{5 !}\left(\sum_{p=1}^{\infty} \frac{1}{w_{p}}\right)+\cdots
$$

\section{Quasi-periodicity of the $\zeta_{N}$ functions}

The aim of this paragraph is to obtain the analogous of the formula of quasiperiodicity of the classical Weierstrass function in the case of a complex variable. It is well-known that there exist two constants $\eta, \eta^{\prime}$, such that:

$$
\zeta\left(z+2 n \omega+2 m \omega^{\prime}\right)=\zeta(z)+2 n \eta+2 m \eta^{\prime}
$$

where $z \in \mathbb{C}, n, m \in \mathbb{Z}$, and the corresponding lattice is generated by $2 \omega$ and $2 \omega^{\prime}$. Moreover, $\eta=\zeta(\omega)$ and $\eta^{\prime}=\zeta\left(\omega^{\prime}\right)$. The first step is to show that the function

$Z_{4}(x, a)=\zeta_{4}(x+a)-\zeta_{4}(a)-(x \mid \nabla) \zeta_{4}(a)-\frac{1}{2 !}(x \mid \nabla)^{2} \zeta_{4}(a)-\frac{1}{3 !}(x \mid$ $\nabla)^{3} \zeta_{4}(a)$ looked as a function of the variable $a$ is holomorphic Cliffordian on $S \oplus V \backslash\left\{w_{m}\right\}_{m=0}^{\infty}$ and also is periodic. In fact, we have to substitute all the terms of this sum by their expansions and observe that all the terms containing polynomial expressions on $a$ are cancelled. The remaining part will satisfy the two required conditions. The computations will be carried on $\zeta_{4}$ denoted for briefness by $\zeta$.

A straightforward calculus gives :

$$
\begin{aligned}
\zeta(x+a) & =(x+a)^{-1}+\sum_{m=1}^{\infty}\left\{\left(x+a-w_{m}\right)^{-1}+w_{m}^{-1}+w_{m}^{-1}(x+a) w_{m}^{-1}\right. \\
& \left.+\left(w_{m}^{-1}(x+a)\right)^{2} w_{m}^{-1}+\left(w_{m}^{-1}(x+a)\right)^{3} w_{m}^{-1}\right\}
\end{aligned}
$$

which can be written as :

$$
\begin{aligned}
& \zeta(x+a)=\sum_{m=0}^{\infty}\left(x+a-w_{m}\right)^{-1}+\sum_{m=1}^{\infty}\left\{w_{m}^{-1}+w_{m}^{-1} x w_{m}^{-1}+w_{m}^{-1} a w_{m}^{-1}+\right. \\
& +\left(w_{m}^{-1} x\right)^{2} w_{m}^{-1}+w_{m}^{-1} x w_{m}^{-1} a w_{m}^{-1}+w_{m}^{-1} a w_{m}^{-1} x w_{m}^{-1}+\left(w_{m}^{-1} a\right)^{2} w_{m}^{-1}+ \\
& +\left(w_{m}^{-1} x\right)^{3} w_{m}^{-1}+\left(w_{m}^{-1} x\right)^{2} w_{m}^{-1} a w_{m}^{-1}+w_{m}^{-1} x w_{m}^{-1} a w_{m}^{-1} x w_{m}^{-1}+ \\
& +w_{m}^{-1} a\left(w_{m}^{-1} x\right)^{2} w_{m}^{-1}+\left(x_{m}^{-1} a\right)^{2} w_{m}^{-1} x w_{m}^{-1}+w_{m}^{-1} a w_{m}^{-1} x w_{m}^{-1} a w_{m}^{-1}+ \\
& \left.+w_{m}^{-1} x\left(x_{m}^{-1} a\right)^{2} w_{m}^{-1}+\left(w_{m}^{-1} a\right)^{3} w_{m}^{-1}\right\}
\end{aligned}
$$


where we have reintroduced in the first sum the power $(x+a)^{-1}$ appearing for $w_{0}=0$.

From this sum we have to substract four other sums :

$\zeta(a)=\sum_{m=0}^{\infty}\left(a-w_{m}\right)^{-1}+\sum_{m=1}^{\infty}\left\{w_{m}^{-1}+w_{m}^{-1} a w_{m}^{-1}+\left(w_{m}^{-1} a\right)^{2} w_{m}^{-1}+\left(w_{m}^{-1} a\right)^{3} w_{m}^{-1}\right\}$

and :

$$
\begin{aligned}
& (x \mid \nabla) \zeta(a)=-\sum_{m=0}^{\infty}\left(a-w_{m}\right)^{-1} x\left(a-w_{m}\right)^{-1}+\sum_{m=1}^{\infty}\left\{w_{m}^{-1} x w_{m}^{-1}+\right. \\
& +w_{m}^{-1} x w_{m}^{-1} a w_{m}^{-1}+w_{m}^{-1} a w_{m}^{-1} x w_{m}^{-1}+\left(w_{m}^{-1} a\right)^{2} w_{m}^{-1} x w_{m}^{-1} \\
& \left.+w_{m}^{-1} a w_{m}^{-1} x w_{m}^{-1} a w_{m}^{-1}+w_{m}^{-1} x\left(w_{m}^{-1} a\right)^{2} w_{m}^{-1}\right\} .
\end{aligned}
$$

Further :

$$
\begin{aligned}
& \frac{1}{2}(x \mid \nabla)^{2} \zeta(a)=\sum_{m=0}^{\infty}\left(\left(a-w_{m}\right)^{-1} x\right)^{2}\left(a-w_{m}\right)^{-1}+\sum_{m=1}^{\infty}\left\{\left(w_{m}^{-1} x\right)^{2} w_{m}^{-1}\right. \\
& \left.\quad+\left(w_{m}^{-1} x\right)^{2} w_{m}^{-1} a w_{m}^{-1}+w_{m}^{-1} x w_{m}^{-1} a w_{m}^{-1} x w_{m}^{-1}+w_{m}^{-1} a\left(w_{m}^{-1} x\right)^{2} w_{m}^{-1}\right\},
\end{aligned}
$$

and finally :

$$
\frac{1}{3 !}(x \mid \nabla)^{3} \zeta(a)=-\sum_{m=0}^{\infty}\left(\left(a-w_{m}\right)^{-1} x\right)^{3}\left(a-w_{m}\right)^{-1}+\sum_{m=1}^{\infty}\left(w_{m}^{-1} x\right)^{3} w_{m}^{-1} .
$$

A careful substraction gives :

$$
\begin{aligned}
& Z_{4}(x, a)=\zeta(x+a)-\zeta(a)-(x \mid \nabla) \zeta(a)-\frac{1}{2 !}(x \mid \nabla)^{2} \zeta(a)-\frac{1}{3 !}(x \mid \nabla)^{3} \zeta(a)= \\
& =\sum_{m=0}^{\infty}\left(x+a-w_{m}\right)^{-1}-\sum_{m=0}^{\infty}\left(a-w_{m}\right)^{-1}+\sum_{m=0}^{\infty}\left(a-w_{m}\right)^{-1} x\left(a-w_{m}\right)^{-1} \\
& -\sum_{m=0}^{\infty}\left(\left(a-w_{m}\right)^{-1} x\right)^{2}\left(a-w_{m}\right)^{-1}+\sum_{m=0}^{\infty}\left(\left(a-w_{m}\right)^{-1} x\right)^{3}\left(a-w_{m}\right)^{-1} .
\end{aligned}
$$

If we consider $Z_{4}(x, a)$ as a function of the variable $a$, because every term in each sum on the right hand side is holomorphic Cliffordian and the convergence is fulffiled then $Z_{4}(x, a)$ is holomorphic Cliffordian on a over $S \oplus V \backslash\left\{w_{n}\right\}_{m=0}^{\infty}$. Remember the lattice $\left\{w_{n}\right\}_{m=0}^{\infty}$ can be seing as $2 \mathbb{Z}^{4} \omega$, all the sums are periodic and so $Z_{4}(x, a)$ is periodic on $a$.

Let us write down this periodicity :

$$
Z_{4}(x, a+2 \omega)=Z_{4}(x, a)
$$


and put here $a=-\omega$, i.e. :

$$
\begin{aligned}
& \zeta(x+\omega)-\zeta(\omega)-(x \mid \nabla) \zeta(\omega)-\frac{1}{2 !}(x \mid \nabla)^{2} \zeta(\omega) \\
& -\frac{1}{3 !}(x \mid \nabla)^{3} \zeta(\omega)=\zeta(x-\omega)-\zeta(-\omega)-(x \mid \nabla) \zeta(-\omega) \\
& -\frac{1}{2 !}(x \mid \nabla)^{2} \zeta(-\omega)-\frac{1}{3 !}(x \mid \nabla)^{3} \zeta(-\omega) .
\end{aligned}
$$

Recall $\zeta$ is odd, so $(x \mid \nabla)^{2} \zeta$ will be also odd and thus :

$$
\zeta(x+\omega)-\zeta(x-\omega)=2 \zeta(\omega)+(x \mid \nabla)^{2} \zeta(\omega) .
$$

Apply now the last formula for $x+\omega$, we get :

$$
\zeta_{4}(x+2 \omega)-\zeta_{4}(x)=2 \zeta_{4}(\omega)+((x+\omega) \mid \nabla)^{2} \zeta_{4}(\omega)
$$

which shows the quasi-periodicity of the $\zeta_{4}$ Weierstrass function. The right hand side is a polynomial on $x$ of degree 2 .

It should be noted that following the same way, we are in a position to get quasi-periodicity formulas for all the $\zeta_{N}$ Weierstrass functions. For $\zeta_{1}$ a direct computation shows that

$$
\zeta_{1}(x+2 \omega)-\zeta_{1}(x)=0 .
$$

For the other cases, $\quad N=2,3,4$, it suffices to erase all the terms which should disappear in the computation of the corresponding $Z_{N}(x, a)$. For instance :

$$
Z_{N}(x, a)=\zeta_{N}(x+a)-\sum_{n=0}^{N-1} \frac{(x \mid \nabla)^{n}}{n !} \zeta_{N}(a), \quad N=2,3,4
$$

Those are $N$-periodic. Writing down the periodicity and taking into account that $\zeta(\omega), \frac{(x \mid \nabla)^{2}}{2 !} \zeta(\omega)$ are odd on $\omega$, one get :

$$
\zeta_{2}(x+2 \omega)-\zeta_{2}(x)=2 \zeta_{2}(\omega)
$$

and

$$
\zeta_{3}(x+2 \omega)-\zeta_{3}(x)=2 \zeta_{3}(\omega)+((x+\omega) \mid \nabla)^{2} \zeta_{3}(\omega) .
$$

Let us introduce a notation fo the right-hand side polynomial of quasiperiodicity as, for example,

$$
\zeta_{N}(x+2 \omega)-\zeta_{N}(x)=\eta_{N}(x, \omega) .
$$


Except in the case $N=1$, when $\eta_{1}$ reduces to the constant zero, $\eta_{N}$ contains terms of the type $2 \frac{((x+\omega) \mid \nabla)^{2 p}}{(2 p) !} \zeta_{N}(\omega)$ with $\left.p \in \llbracket 0,\left[\frac{N+1}{2}\right]-1\right]$ when $N \geq 2$. So that :

$$
\eta_{N}(x, \omega)=2 \sum_{p=0}^{\left[\frac{N+1}{2}\right]-1} \frac{((x+\omega) \mid \nabla)^{2 p}}{(2 p) !} \zeta_{N}(\omega)
$$

\section{Some other formulas for the $\zeta_{4}$ function}

The property of quasi-periodicity of $\zeta_{4}$, i.e. formula $(5)$ of $\S 4$, allows us to obtain other formulas.

First, let us introduce :

$$
\begin{aligned}
\eta(x, \omega) & =2 \zeta_{4}(\omega)+\left((x+\omega) \mid \nabla_{\omega}\right)^{2} \zeta_{4}(\omega) \\
& =2\left(1+\left((x+\omega) \mid \nabla_{\omega}\right)^{2}\right) \zeta_{4}(\omega),
\end{aligned}
$$

so that the quasi-periodicity of $\zeta_{4}$ could be written as :

$$
\zeta_{4}(x+2 \omega)=\zeta_{4}(x)+\eta(x, \omega)
$$

Look now at some elementary properties of $\eta(x, \omega)$ :

(i) $\eta$ is a polynomial on $x$ of degree 2 over $\mathbb{R}_{0,3}$.

Take $\zeta_{4}(x)$. We can write on one hand :

$$
\zeta_{4}(x)=\zeta_{4}(x-2 \omega+2 \omega)=\zeta_{4}(x-2 \omega)+\eta(x, \omega)
$$

so that $\zeta_{4}(x-2 \omega)=\zeta_{4}(x)-\eta(x, \omega)$. But, on the other hand

$$
\zeta_{4}(x-2 \omega)=\zeta_{4}(x)+\eta(x,-\omega) .
$$

In such a way we get :

(ii) $\eta(x,-\omega)=-\eta(x, \omega)$, which means that $\eta$ is a even function on its second variable.

The argument below gives also :

$$
\zeta_{4}(x-2 \omega)=\zeta_{4}(x)-\eta(x, \omega) .
$$


Now compute :

$$
\begin{aligned}
\eta(-x, \omega) & =2 \zeta(\omega)+\left((-x+\omega) \mid \nabla_{\omega}\right)^{2} \zeta(\omega) \\
& =-2 \zeta(-\omega)-\left((x-\omega) \mid \nabla_{\omega}\right)^{2} \zeta(-\omega) \\
& =-\eta(x,-\omega)=\eta(x, \omega)
\end{aligned}
$$

which shows :

(iii) $\eta$ is an even function of its first variable $x$.

As a direct consequence of (ii) and (iii) we get :

(iv) $\eta(-x,-\omega)=-\eta(x, \omega)$.

The last result could be obtained also by a direct computation carried on the defining expression of $\eta$.

Developping the defining expression for $\eta$ we have :

$$
\begin{aligned}
\eta(x, \omega) & =2 \zeta(\omega)+\left(\omega \mid \nabla_{\omega}\right)^{2} \zeta(\omega) \\
& +2\left(x \mid \nabla_{\omega}\right)\left(\omega \mid \nabla_{\omega}\right) \zeta(\omega) \\
& +\left(x \mid \nabla_{\omega}\right)^{2} \zeta(\omega),
\end{aligned}
$$

in which we have to erase those terms which are zero, i.e. those in which appears $\left(\omega \mid \nabla_{\omega}\right) \zeta(\omega)$ so :

(v) $\eta(x, \omega)=2 \zeta(\omega)+\left(x \mid \nabla_{\omega}\right)^{2} \zeta(\omega)$.

In the last, set $x=\omega$. Thus we have :

(vi) $\eta(\omega, \omega)=2 \zeta(\omega)$.

Remark (vi) could be obtained directly from (6) setting $x=-\omega$, or from (7) with $x=\omega$.

We will end this paragraph with the following : take two half-periods $\omega^{1}$ and $\omega^{2}$. Set $\omega^{12}$ such that

$$
\omega^{1}+\omega^{2}+\omega^{12}=0
$$

Apply formula (7) on :

$$
\begin{aligned}
& \zeta_{4}\left(x+2 \omega^{12}\right)=\zeta_{4}\left(x-2 \omega^{1}-2 \omega^{2}\right)= \\
& =\zeta_{4}\left(x-2 \omega^{1}\right)-\eta\left(x, \omega^{2}\right)= \\
& =\zeta_{4}(x)-\eta\left(x, \omega^{1}\right)-\eta\left(x, \omega^{2}\right) .
\end{aligned}
$$


On the other hand, from (6) we have :

$$
\zeta_{4}\left(x+2 \omega^{12}\right)=\zeta_{4}(x)+\eta\left(x, \omega^{12}\right)
$$

so that :

$$
\eta\left(x, \omega^{1}\right)+\eta\left(x, \omega^{2}\right)+\eta\left(x, \omega^{12}\right)=0
$$

\section{$\S$ 6. Cliffordian analogues of the Weierstrass $\mathcal{P}$ function}

Remember the classical situation of elliptic functions. Once we have constructed the $\zeta$ function, which is an odd function, quasi-periodic on $2 \omega_{1}$ and $2 \omega_{2}$, and which is defined as :

$$
\zeta(z)=\frac{1}{z}+\sum_{p=1}^{\infty}\left\{\frac{1}{z-w_{p}}+\frac{1}{w_{p}}+\frac{z}{x_{p}^{2}}\right\},
$$

where the set $\left(2 \mathbb{Z} \omega_{1}+2 \mathbb{Z} \omega_{2}\right) \backslash\{(0,0)\}$ has been rearranged in a set denoted by $\left\{w_{p}\right\}, p \in \mathbb{N}^{*}$, then, in order to introduce an authentic 2-periodic function, we need to derive $\zeta$ :

$$
\zeta^{\prime}(z)=\frac{d}{d z} \zeta(z)=\frac{\partial}{\partial x} \zeta(z)=-i \frac{\partial}{\partial y} \zeta(z)
$$

The tradition demands to set :

$$
\mathcal{P}(z)=-\zeta^{\prime}(z)
$$

so that :

$$
\mathcal{P}(z)=\frac{1}{z^{2}}+\sum_{p=1}^{\infty}\left\{\frac{1}{\left(z-w_{p}\right)^{2}}-\frac{1}{w_{p}^{2}}\right\}
$$

Obviously, $\mathcal{P}$ is an even function. One want to show that $\mathcal{P}$ is a 2-periodic function. For this purpose there is a traditionnal argument based on the fact that obviously $\mathcal{P}^{\prime}$ is a 2-periodic function and because of the parity of $\mathcal{P}$ one can conclude, by an integration, that $\mathcal{P}$ is also 2-periodic.

In our situation, we start with the $\zeta_{4}$ function :

$$
\zeta_{4}(x)=x^{-1}+\sum_{p=1}^{\infty}\left\{\left(x-w_{p}\right)^{-1}+\sum_{\mu=0}^{3}\left(w_{p}^{-1} x\right)^{\mu} w_{p}^{-1}\right\} .
$$

Here, the possibilities of derivations are larger than in the previous case, so the candidates for analogues of the $\mathcal{P}$ function are numerous. 
It would be possible to restrict us on a canonical way of derivation, just on the real axis, namely on $\frac{\partial}{\partial x_{0}}$. Taking into account we need three successive derivations, one of the $\mathcal{P}_{4}$ functions would be :

$$
\begin{aligned}
\mathcal{P}_{4}(x) & =-\frac{1}{3 !} \frac{\partial^{3}}{\partial x_{0}^{3}} \zeta_{4}(x)= \\
& =x^{-4}+\sum_{p=1}^{\infty}\left\{\left(x-w_{p}\right)^{-4}-w_{p}^{-4}\right\} .
\end{aligned}
$$

In fact, the right notation for the last function should be $\mathcal{P}_{(3,0,0,0)}(x)$, in which we indicate the used derivations.

Generally, we have $20 \mathcal{P}$-functions coming from $\left(e_{i} \mid \nabla_{x}\right)^{3} \zeta_{4}(x), i=$ $0,1,2,3$. In the same way we introduced the notation for the holomorphic Cliffordian polynomials in $\S 1$, if $\alpha=\left(\alpha_{0}, \alpha_{1}, \alpha_{2}, \alpha_{3}\right)$ is a multiindex in $\mathbb{N}^{4}$ with lenght $|\alpha|=3$, we can look at the $\mathcal{P}_{\alpha}(x)$ functions.

For example :

$$
\begin{aligned}
& \mathcal{P}_{(3,0,0,0)}(x)=\left(e_{0} \mid \nabla_{x}\right)^{3} \zeta_{4}(x), \\
& \mathcal{P}_{(2,0,1,0)}(x)=\left(e_{0} \mid \nabla_{x}\right)^{2}\left(e_{2} \mid \nabla_{x}\right) \zeta_{4}(x), \\
& \mathcal{P}_{(0,1,1,1)}(x)=\left(e_{1} \mid \nabla_{x}\right)\left(e_{2} \mid \nabla_{x}\right)\left(e_{3} \mid \nabla_{x}\right) \zeta_{4}(x)
\end{aligned}
$$

Now let us show that the $\mathcal{P}_{\alpha}$ functions are 4-periodics. For this we will observe that, again by derivation, one gets :

$$
\left(e_{i} \mid \nabla_{x}\right) \mathcal{P}_{\alpha}(x+2 \omega)=\left(e_{i} \mid \nabla_{x}\right) \mathcal{P}_{\alpha}(x) .
$$

Here $i=0,1,2,3, \quad \alpha=\left(\alpha_{0}, \alpha_{1}, \alpha_{2}, \alpha_{3}\right),|\alpha|=3$.

Integrating this four relations respectively in the directions $e_{0}, e_{1}, e_{2}, e_{3}$, one deduces :

$$
\mathcal{P}_{\alpha}(x+2 \omega)=\mathcal{P}_{\alpha}(x)+C_{i}\left(x_{0}, \ldots, \hat{x}_{i}, \ldots, x_{3}\right)
$$

for $i=0,1,2,3$, where $C_{i}\left(x_{0}, \ldots, \hat{x}_{i}, \ldots, x_{3}\right)$ designe "constants" depending on the mentioned variables. By successive substractions we can prove the independance of the variables of the constants. So for every $x \in S \oplus V$, one has :

$$
\mathcal{P}_{\alpha}(x+2 \omega)=\mathcal{P}_{\alpha}(x)+C
$$

with a constant $C$. Setting $x=-\omega$, we have :

$$
\mathcal{P}_{\alpha}(\omega)=\mathcal{P}_{\alpha}(-\omega)+C
$$


from which we deduce $C=0$ because of the parity of $\mathcal{P}_{\alpha}$. Thus the 4-periodicity of $P_{\alpha}$ was proved.

All the $\mathcal{P}_{\alpha}$ functions are elliptic Cliffordian with pointwise poles of order 4 at the vertices of the latice $2 \mathbb{Z}^{4} \omega$.

\section{$\S 7$. On the sets of the zeros and the poles of $D_{0} \mathcal{P}_{0}$}

Here $D_{0}=\frac{\partial}{\partial x_{0}}$ and $\mathcal{P}_{0}$ is an abreviated notation for $\mathcal{P}_{(3,0,0,0)}$.

According to (8) it is clear that the set of the poles of $D_{0} \mathcal{P}_{0}$ is $2 \mathbb{Z}^{4} \omega$.

Let now restrict us in an elementary cell in $\mathbb{R}^{4}$ which is nothing else than a hypercube spanned over four linearly independant paravectors $2 \omega_{1}, 2 \omega_{2}, 2 \omega_{3}, 2 \omega_{4}$ (in fact four $\mathbb{R}$-independant vectors in $\mathbb{R}^{4}$ ). Thus, the only pole of $D_{0} P_{0}$ in this cell is a pole of order 5 at the origin.

Try now to find the zeros of $D_{0} \mathcal{P}_{0}$. Remember $D_{0} \mathcal{P}_{0}$ is 4-periodic and odd. So :

$$
D_{0} \mathcal{P}_{0}(x+2 \omega)=D_{0} \mathcal{P}_{0}(x) .
$$

Set $x=-\omega$, one deduces

$$
D_{0} \mathcal{P}_{0}(\omega)=D_{0} \mathcal{P}_{0}(-\omega)=-D_{0} \mathcal{P}_{0}(\omega)
$$

from which :

$$
D_{0} \mathcal{P}_{0}(\omega)=0
$$

that means $D_{0} \mathcal{P}_{0}$ vanishes at all the half-periods $\omega_{1}, \omega_{2}, \omega_{3}, \omega_{4}$ and their linear combinations by 2 , by 3 elements and finally at the vertex $\omega_{1}+\omega_{2}+$ $\omega_{3}+\omega_{4}$ that is at all the vertices (excepting 0 ) of the hypercube spanned over $\omega_{1}, \omega_{2}, \omega_{3}, \omega_{4}$. The number of this vertices is $2^{4}-1=15$.

At this stage we do not have guarantees this are the only zeros of $D_{0} \mathcal{P}_{0}$. The result is only that, concerning the 4-periodic function $D_{0} \mathcal{P}_{0}$,

$$
\mathcal{Z} \geq 3 P
$$

where $\mathcal{Z}$ means the set of its zeros and $P$ the set of his poles, as usually, taking into account the multiplicities.

Conjecture. Is is true that in an elementary cell of the 4-periodic lattice, every 4-periodic function $f$ satisfies

$$
\mathcal{Z}_{f}=3 P_{f} ?
$$




\section{Bibliographie}

[1] F. BRACKS, R. DELANGHE, F. SOMMEN - Clifford analysis, ; Pitman, (1982).

[2] C.A. DEAVORS - The quaternion calculus ; Am. Math. Monthly. (1973), 995-1008.

[3] R. DELANGHE, F. SOMMEN, V. SOUCĚK - Clifford Algebra and Spinor-valued functions ; Kluwer Academic Publishers.

[4] R. FUETER - Die Funktionnentheorie der Differentialgleichungen $\Delta u=0$ und $\Delta \Delta u=0$ mit vier reellen Variablen. ; Comment Math. Helv 7 (1935), 307-330.

[5] R. FUETER - Uber die analytische Darstellung der regularen Funktionen einer Quaternionenvariabelen ; Comm. Math. Helv.8 (1936), 371-378.

[6] G. LAVILLE - Une famille de solutions de l'équation de Dirac avec champ électromagnétique quelconque ; C.R. Acad. Sci. Paris t. 296 (1983), 1029-1032.

[7] G. LAVILLE - Sur l'équation de Dirac avec champ électromagnétique quelconque ; Lectures Notes in Math. 1165, Springer-Verlag (1985), 130-149.

[8] G. LAVILLE, I. RAMADANOFF - Fonctions holomorphes Cliffordiennes ; C.R. Acad, Sc. Paris, 326, série I (1998), 307-310.

[9] G. LAVILLE, I. RAMADANOFF - Holomorphic Cliffordian Functions ; Advances in Applied Clifford Algebras, 8, n² (1998), 323-340.

[10] H. MALONEK - Powers series representation for monogenic functions in $\mathbb{R}^{n+1}$ based on a permutational product ; Complex variables, vol 15 (1990), 181-191.

[11] L. PERNAS - Holomorphie quaternionienne ; Advances in Applied Clifford Algebras, 8, n² (1998), 283-298.

[12] P.L. WALKER - Elliptic Functions ; J. Wiley and Sons (1996).

UPRES-A 6081 Département de Mathématiques

Université de Caen

14032 CAEN Cedex France

glaville@math.unicaen.fr

rama@math.unicaen.fr 